\title{
DMBA-Induced Mammary Pathologies are Angiogenic In Vivo and In Vitro
}

\author{
Sue C. Heffelfinger, Robin B. Gear, Kevin Taylor, Mary Ann Miller, \\ Joanne Schneider, Kathy LaDow, and David Warshawsky \\ Departments of Pathology and Laboratory Medicine (SCH, RBG, KT, MAM) and Environmental Health (JS, KL, DW), \\ University of Cincinnati, Cincinnati, Ohio
}

SUMMARY: We have previously shown that human pre-invasive diseases of the breast are angiogenic. In addition, normal epithelium from women with coincident or subsequent invasive breast cancer is more vascular than normal epithelium from women with no breast cancer. To develop a model in which to study the regulation of angiogenesis in pre-invasive mammary pathologies, we examined 7,12-dimethylbenz[a]anthracene (DMBA)-induced rat mammary tissues for the presence of neovascularization in pre-invasive histopathologies. These studies included morphometric analysis of tissue vascularity in pre-invasive lesions. In addition, we isolated fresh tumors and histologically normal epithelium (organoids) from DMBA or vehicle-treated control rats to test their ability to induce endothelial cell tubule formation in vitro. Finally, we examined tumors for their ability to produce vascular endothelial cell growth factor. The morphometric studies documented that with epithelial progression, the ability of individual cells to elicit angiogenesis increases. The in vitro studies showed that isolated tumors from these animals stimulate angiogenesis. Furthermore, normal epithelium from DMBA-treated rats is more angiogenic than epithelium from control animals. Finally, DMBA-induced tumors produce vascular endothelial growth factor (VEGF) mRNA, therefore, DMBA-induced mammary tumorigenesis is one model in which to test the dependency of progression on angiogenesis. (Lab Invest 2000, 80:485-492).

A Ithough it is well established that invasive tumors require neovascularization for continued growth, only recently have pre-invasive pathologies been shown to elicit the formation of new vessels (Brem et al, 1977; Folkman et al, 1989; Fregene et al, 1994; Kandel et al, 1991; Ottinetti and Sapino, 1988; Smith-McCune and Weidner, 1994). Because of the prognostic importance of angiogenesis in invasive breast tumors, we examined the vascularity of pre-invasive breast pathologies, ie, lesions that confer a statistical risk of subsequently developing invasive disease (Dupont and Page, 1985). Our studies showed that tissue vascularity increases with disease progression and the degree of vascularity correlates with the histologic features that confer risk (Heffelfinger et al, 1996). Furthermore, even histologically normal epithelium in women who had or would shortly acquire invasive disease was more vascular than normal epithelium from women without breast cancer (Heffelfinger et al, 1996). These studies suggest that disease progression from normal epithelium to invasion depends upon induction of a new blood supply. However, to functionally test this question requires an animal model system.

Received October 25, 1999.

Supported by the Ruth Lyons Foundation and The Greater Cincinnati Foundation

Address reprint requests to: Dr. S.C. Heffelfinger, Department of Pathology and Laboratory Medicine, PO Box 670529, University of Cincinnati, Cincinnati, Ohio 45267-0529. Fax: 513-558-2141; Email: sue.heffelfinger@uc.edu
Carcinogen-induced mammary tumors in rodents have been widely used to study the process of carcinogenesis. Features of this process that make the model comparable to human disease include similarities in histologic progression and hormone dependence (Russo and Russo, 1996). We asked whether neovascularization with progression might be a third feature. Indeed, some very early studies indicated that murine hyperplastic alveolar nodules could induce a vascular supply in the rabbit iris assay system (Gimbrone and Gullino, 1976). To expand upon this work we performed a detailed examination of the vascularity of 7,12dimethylbenz[a]anthracene (DMBA) -induced rat mammary pathologies.

In the DMBA rat model, cellular changes occur within 24 hours of carcinogen exposure (Russo and Russo, 1987). Depending upon the state of development of the mammary tissue at the time of initiation, multiple histologic pathways of tumorigenesis can be identified (Russo and Russo, 1987). Carcinogen exposure to mammary lobules results in the formation of lobular hyperplasia. Less mature mammary tissues containing alveolar buds progress to hyperplastic alveolar nodules (HP) and tubular adenomas (TA), a form of non-invasive mammary pathology. Finally, immature mammary tissues containing terminal end buds progress to intraductal hyperplasia (IDP), carcinoma in situ (CIS), and finally invasive carcinoma (INV). Because rodents have dissynchronous mammary development, more than one pathway may be identified in an individual animal after carcinogen exposure. We hypothesized that the vascularity of each type of pathology would reflect the propen- 
sity for that lesion to progress to invasive disease. Therefore, we would predict that HP, TA, IDP, and CIS would be more vascular than normal epithelium, and that HP and TA would be less vascular than IDP or CIS. Finally, if these tumors contained an increased vascularity, we predicted that they would be angiogenic in vitro. Here we report that DMBA-induced mammary pathologies induce a vascular supply early in disease progression. In addition, this neovascularization is, at least in part, due to induction of angiogenesis.

\section{Results}

Two experiments were performed in which rats were treated with DMBA or vehicle (sesame oil) and followed for mammary tumor production. In the first experiment, at 3 months post-DMBA, 75\% (3/4) of animals had both CIS and INV tumors. One of five animals originally treated with DMBA died 1 week post-DMBA of aspiration pneumonia. Control animals, treated with sesame oil, were all free of CIS and INV, as well as the precursor pathologies. To confirm these data we repeated the study with three animals per group. At 3 months post-DMBA 100\% (3 of 3) of animals had CIS and 2 of 3 had INV. Control animals had no pathology (0 of 3 ). Morphometric analysis of the normal and pathologic lesions from the two experiments gave similar results. The figures below show the results from the first experiment.

In our initial examination of the tissues, we determined the total vascularity per $10 \times$ objective field, with the stipulation that only one type of epithelium be present within the field. IDP occur in clusters and thus were easy to accommodate to this methodology. HP, on the other hand, occur singly, and therefore, all HP data are the result of individual "lobule" analysis (see below). All animals were given bromo-deoxyuridine (BrdU) 24 hours before they were killed. Morphometric analyses were performed on histologic sections immunolabeled for both von Willebrand factor (brown) and BrdU incorporation (blue). Figure 1 shows an example of the vascularity and degree of vessel proliferation seen in a DMBA-induced tumor. As expected clusters of tumor cells show considerable BrdU incorporation (arrowheads). However, the vasculature also contains numerous BrdU-positive endothelial cells (arrows).

Figure 2 a shows the analysis of the non-BrdUpositive vascularity per $10 \times$ field for normal epithelium from sesame oil and DMBA-treated animals, and IDP, CIS, and INV, which are all from DMBA-treated animals. Note that the vascularity for IDP, CIS, and INV are significantly greater than for normal epithelium from either the control or DMBA-treated rats $(p<$ 0.001). In addition, the vascularity of histologically normal epithelium is similar between the sesame oil and DMBA-treated rats. Because BrdU-positive vascularity is a better predictor of neovascularization, we reanalyzed these data using only BrdU-positive vessels (Fig. 2b). Note the significant differences, above, are maintained when one considers either total vascularity or BrdU-positive vessels. However, the BrdU-

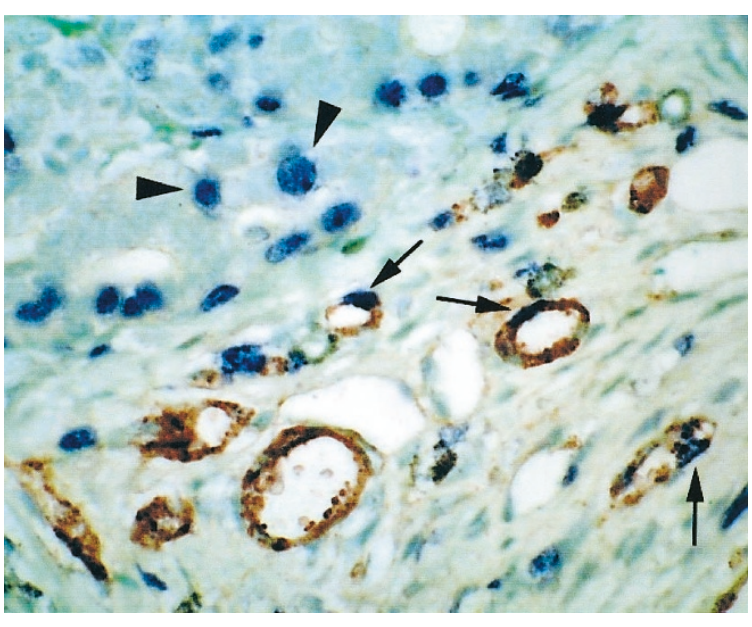

Figure 1.

shows a histologic section of 7,12-dimethylbenz[a]anthracene (DMBA)induced carcinoma in situ (CIS) stained for immunoreactivity to von Willebrand factor (brown), which identifies vessels, and BrdU incorporation (blue), which identifies cells undergoing DNA synthesis in the 24 hours immediately before the rats were killed. Arrowheads indicate BrdU-positive nuclei in the epithelium. Arrows denote BrdU-positive endothelium. (Original magnification, $\times 60$ )

positive vascularity of normal epithelium from sesame oil-treated animals is almost zero. By this analysis the normal epithelium from DMBA-treated rats is significantly more vascular than the normal epithelium from sesame oil-treated animals $(p<0.05)$. While these data indicate that the tissue in general has increased vascularity with disease progression, the epithelial areas also significantly increase with disease progression. One could interpret these data as confirming that with any tissue growth, an increase in vascularity must occur. However, if the angiogenic potential of individual epithelial cells were increased with progression, one would expect that vascular/epithelial area would also increase. Figure 3 shows the BrdU-positive vascularity normalized to epithelial area. By this analysis epithelium within IDP, CIS, and INV induce more neovascularization per unit area than normal epithelium from control animals ( $p<0.001)$. Furthermore, histologically normal epithelium from DMBA-treated rats also induces greater neovascularization than normal epithelium from the sesame oil controls ( $p<$ $0.05)$. In addition, IDP and INV are more vascular than normal epithelium from DMBA-treated rats $(p<0.05)$. These data suggest that the initiation of the angiogenic phenotype begins before evident histopathology in the epithelium and increases with disease progression to IDP. After the epithelial expansion seen with CIS, there is a drop in the relative vascular area/epithelial area. Finally, there is an additional vascular expansion with the onset of invasion.

To gain more information about the vascularity associated with individual lobules, we examined BrdU-positive vascularity adjacent to each epithelial region ( $\leq 50 \mu \mathrm{m}$ of normal epithelium, HP, and IDP and $\leq 350 \mu \mathrm{m}$ of $\mathrm{CIS}$ and INV). The number of units examined in the first experiment are as follows, with the number examined in the second experiment shown in parentheses: 8 (12) HP, 10 (8) IDP, 7 (6) CIS, 


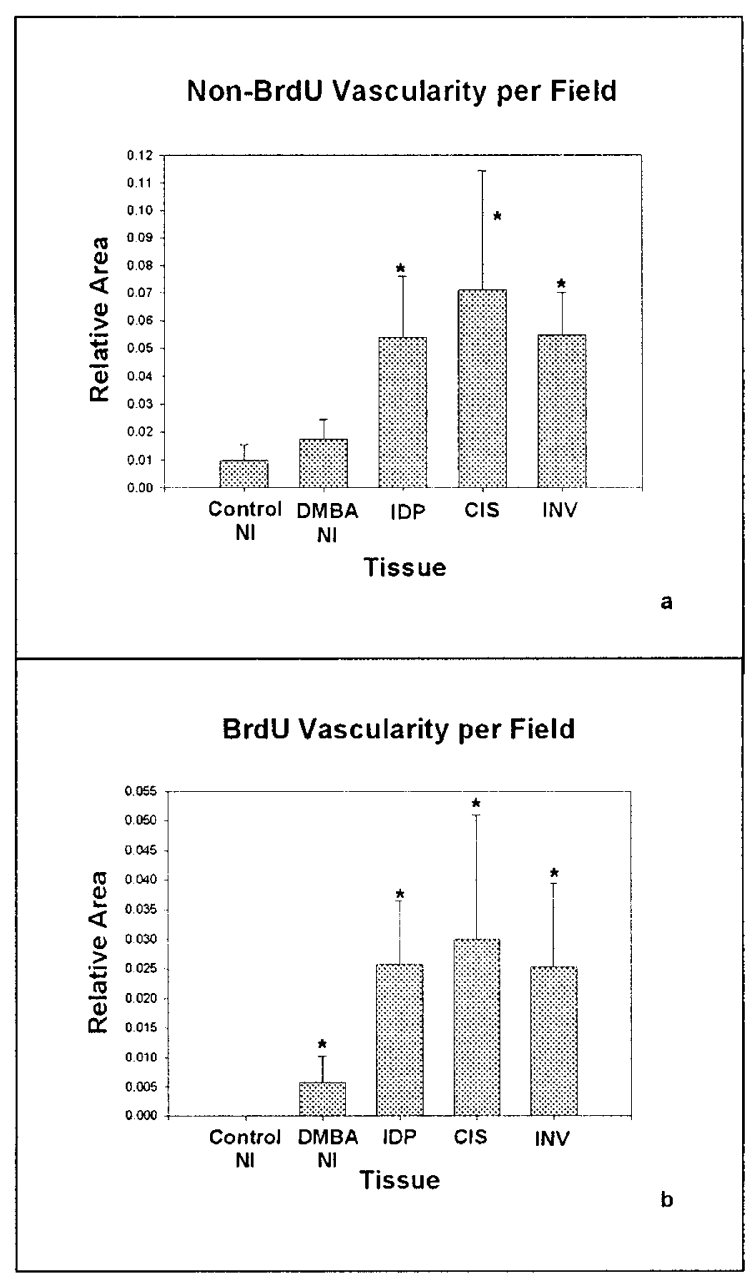

\section{Figure 2.}

shows vascularity data analyzed by $10 \times$ objective field. 2a shows the non-BrdU-positive vascularity per $10 \times$ objective field (Relative Area) containing exclusively either normal epithelium from control (Control NI) or DMBA(DMBA NI) treated rats, intraductal hyperplasia (IDP), CIS, or invasive carcinoma (INV). Plotted are the mean \pm the standard deviation for each group. ${ }^{*}$ indicates those groups with means statistically greater than the mean for Control $\mathrm{NI}(p<0.001)$. These samples are also statistically greater than DMBA NI $(p<0.05)$. $2 \mathrm{~b}$ is the same data analyzed exclusively with BrdU-positive vessels. Note that here the DMBA NI tissue is more vascular than Control $\mathrm{NI}(p<0.05)$. This is primarily due to the fact that vessels from Control NI tissue were almost never BrdU-positive.

and 7 (6) INV. Considerably more normal lobules were examined due to the scarce vascularity in these tissues (221 [106] from control rats and 281 [69] from DMBA-treated rats, derived from 20 and $3410 \times$ fields, respectively). These results are similar to those that utilized entire fields. Figure 4 shows that the ratio of vessel area to epithelial area is greater in IDP, CIS, and INV relative to normal epithelium from DMBAtreated rats $(p<0.05)$. In addition, normal epithelium from DMBA-treated rats is more vascular than that from control rats $(p<0.05)$, consistent with our previous total field analysis. Finally, HP vascularity is not significantly different from normal epithelium from DMBA treated rats but is significantly greater than normal epithelium from control rats $(p<0.05)$.

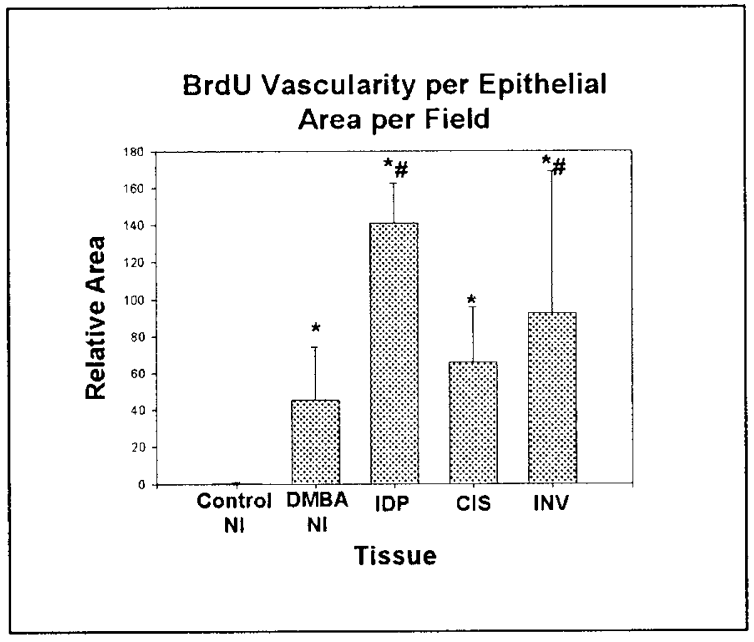

Figure 3.

shows the BrdU-positive vascularity corrected for epithelial area within the field. Plotted are the mean \pm the standard deviation for each group. Note that DMBA NI, IDP, CIS, and INV are all more vascular than normal epithelium from vehicle-treated control animals $\left(^{*}=p<0.05\right.$ ). IDP and INV are also more vascular than DMBA NL ( $\#=p<0.05)$. IDP are especially vascular, despite the epithelial area being smaller than most normal lobules. On the other hand, $\mathrm{CIS}$ and INV are macroscopic structures that occupy multiple $10 \times$ fields.

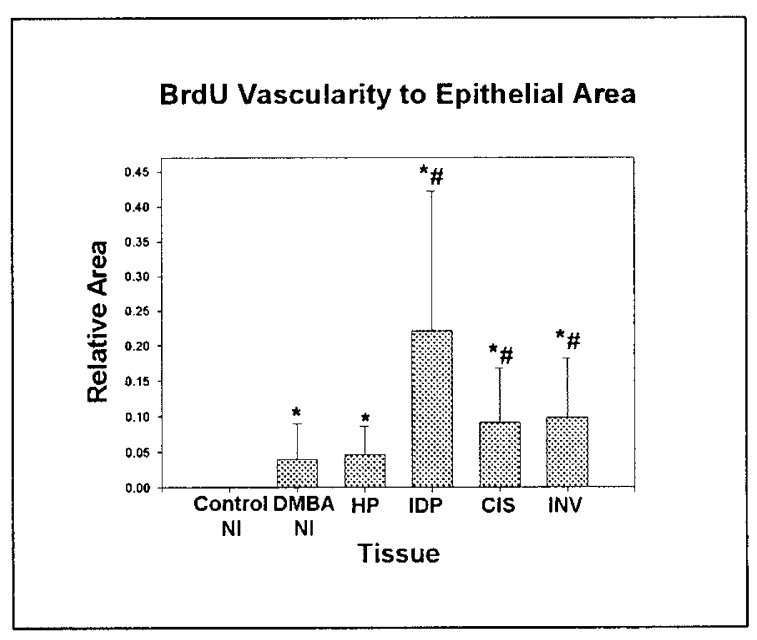

Figure 4.

shows BrdU-positive vascular areas obtained from tissue immediately adjacent to the epithelium. For normal epithelium, hyperplastic alveolar nodules (HP), and IDP the areas were confined to within $50 \mu \mathrm{m}$ of the epithelial basement membrane. For CIS and INV, the areas were $350 \mu \mathrm{m}$. Plotted are the mean \pm the standard deviation for each group. As seen in the previous analyses, all tissues from DMBA-treated animals are more vascular than tissues from vehicle-treated control animals $\left({ }^{*}=p<0.001\right)$. In addition, $\mathrm{HP}$ are also more vascular than Control NI $(p<0.05)$. Finally, IDP, CIS and INV are more vascular than DMBA NL $(\#=p<0.05)$.

The fact that vessel proliferation was unique to the DMBA-induced pathologies suggests that the neovascularization in these tissues was due to angiogenesis. If the tumors were angiogenic, then we predicted that they would be capable of inducing endothelial tubule formation in vitro. Figure 5 shows the results from experiments in which isolated epithelial organoids from control and DMBA-treated rats or CIS from DMBA-treated rats were tested for their ability to 


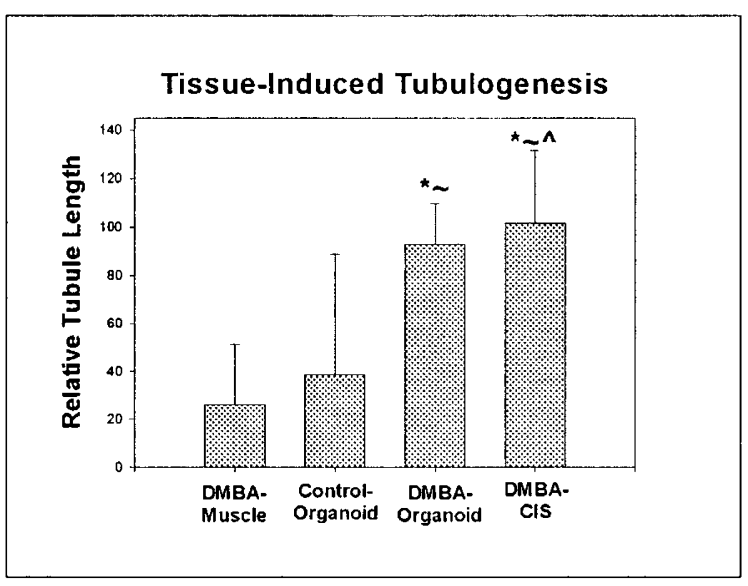

\section{Figure 5.}

shows the degree to which rat tissues are able to induce tubule formation by HUVEC relative to media alone. In this study, skeletal muscle, CIS, and isolated normal epithelial organoids from control or DMBA-treated rats were placed on top of a collagen sandwich in which HUVECS had been plated. Nine hours later the total tubular length for each well was determined by morphometry. Shown are the mean relative tubule length compared with media with no growth factor $(0 \%)$ and $50 \mathrm{n} / \mathrm{ml}$ basic fibroblast growth factor (bFGF) (100\%) from four CIS tissues, four independent organoid preparations from vehicle-treated control rats, three preparations from DMBA-treated rats, and muscle derived from two DMBA-treated rats. * indicates those tissues that induce significantly more tubule formation than media with no growth factor, $\sim$ indicates statistical differences compared with muscle from DMBA-treated rats, and ^ indicates statistical differences compared with epithelial organoids from sesame oiltreated rats $(p<0.05)$.

induce endothelial tubules in a collagen sandwich assay. Shown are the mean and standard deviation of several isolates as discussed in the legend. These pooled data are normalized to both media alone (negative control: set at $0 \%$ ) and media containing 50 $\mathrm{ng} / \mathrm{ml}$ basic fibroblast growth factor (positive control: set at $100 \%$ activity). Similar mean tubular lengths are seen with $50 \mathrm{ng} / \mathrm{ml}$ basic fibroblast growth factor (bFGF) and $100 \mathrm{ng} / \mathrm{ml}$ vascular endothelial growth factor (VEGF) (data not shown). By this assay both CIS and normal epithelium from DMBA-treated rats are significantly angiogenic in vitro, as compared with skeletal muscle from DMBA-treated rats or normal epithelium from sesame oil control animals $(p<0.05)$. Skeletal muscle from vehicle or DMBA-treated rats induce similar levels of tubule formation (not shown).

Because VEGF is known to be important in the neovascularization of many different tumors, we examined INV and CIS, as well as organoids from DMBA-treated and control rats, for the presence of VEGF mRNA by RT-PCR. Primers were chosen to span all reported splice variants. Figure 6 shows the results of these studies in which VEGF mRNA was identified in seven of seven tumors tested [4 CIS (lanes $b$ to e] and 3 INV [lanes $f$ to $h$ ]). Lane a shows the molecular size standards. Lane $\mathrm{i}$ is the PCR negative control without template. Predicted RT-PCR product sizes for VEGF121 (432 bp), VEGF 165 (564 bp), and VEGF189 (636 bp) are seen in all tumor lanes. RNA integrity and cDNA production were controlled for by RT-PCR of rat ribosomal protein L19 (not shown). Epithelial isolates from four vehicle control rats and 6

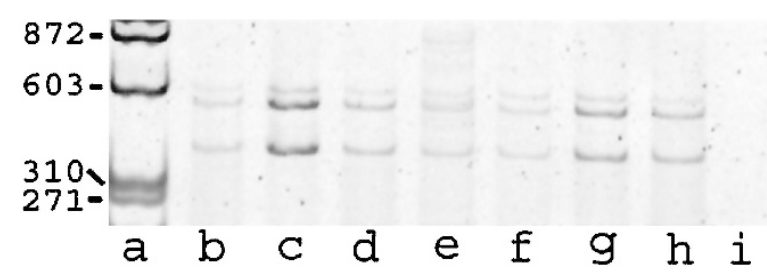

Figure 6.

shows the results of RT-PCR analysis of tumor tissues for VEGF. Molecular size markers are in Lane $a$. Lanes $b$ to e are from CIS and lanes $f$ to $h$, from INV. Lane i is a no-template negative control. The three vascular endothelial growth factor (VEGF) splice variants previously reported by others (VEGF 121, 164, and 189) are seen in all tumors tested.

DMBA-treated rats showed a mixed picture. Two of four vehicle control samples were positive for VEGF mRNA, whereas only one of six samples from DMBAtreated rats were positive. All data were confirmed by repeat RT-PCR analysis from the original RNA. When VEGF mRNA was identified, the presence and distribution of isozymes was the same as in the tumors (not shown).

\section{Discussion}

The majority of studies utilizing morphometric analysis of tumor vascularity report vessel number and/or area per high-powered field. Note in these cases, usually the entire field is composed of tumor. Assessing the vascularity of non-invasive pathologies has not been standardized. Furthermore, theoretical concerns regarding diffusion distances within a tissue surrounding a given epithelial structure are not readily determined. Indeed, it is not clear whether the epithelium alone is responsible for the production of angiogenic agents in all circumstances. Therefore, we assessed vascularity in a number of different ways. First, all of the vessels in a $10 \times$ objective field containing one type of epithelium were measured. This could be accomplished for all types of epithelium, except for hyperplasia, which in all cases was closely associated with normal epithelium and never found in sufficient clusters to constitute a field. Second, epithelium-associated vessels were identified. Initially this was done empirically. Because rodent mammary glands are embedded in adipose tissue with very little extralobular stroma, this was a fairly easy and reproducible assessment to make, being essentially the same regardless of the assessor. Having ascertained by observation which vessels were associated with an epithelial area, we went back to these same areas and measured the distances involved. For the small epithelial areas (normal, HP, and IDP), in all cases this distance was less than 50 $\mu \mathrm{m}$ from the epithelial border. However, for the large tumors, which produced a more abundant stromal reaction, "associated" vessels could be as far as 350 $\mu \mathrm{m}$ from the epithelial edge. Although $100 \mu \mathrm{m}$ is considered by some to be the diffusion limit, we felt that all of the vessels within this larger area should be included, because the vessels greater than $300 \mu \mathrm{m}$ from the epithelium were often BrdU positive. Using a $350 \mu \mathrm{m}$ limit around the normal lobules, HP and IDP 
would result in either no increase in vessel number, or more frequently, the inclusion of another epithelial unit. On the other hand, limiting the area around CIS and INV to $100 \mu \mathrm{m}$, would arbitrarily transect clusters of dense vascularity. Finally, we do not know whether the epithelium alone is responsible for the production of angiogenic agents. If the epithelium were to induce a stromal response which is angiogenic, then one could legitimately reason that these distant vessels were in response to the epithelial pathology. Because we do not know the mechanisms involved, we chose to do our measurements both ways.

The result of these analyses is that by both total field and individual lobule/lesion analysis IDP, CIS, and INV are more vascular than normal epithelium. However, one should not conclude that neovascularization begins with the formation of IDP. Indeed, histologically normal epithelium from DMBA-treated animals is statistically more vascular than the same tissues from sesame oil controls. Note that these changes are being measured 3 months post-DMBA exposure, indicating that profound changes in histologically normal epithelium can be identified long after initiation. This is consistent with what we find in archival tissues from women, in that normal epithelium from cancerous breasts is more vascular than normal epithelium from non-cancerous breasts (Heffelfinger et al, 1996). In fact, a similar observation was made by Jensen et al (1982) years ago. We believe that this neovascularization is driven by genetic changes that lead to an angiogenic phenotype without additional morphologic changes (at least which are obvious to us). In women, several investigators have now identified genetic alterations in normal epithelium adjacent to invasive disease, which may help explain these observations (Deng et al, 1996; Larson et al, 1998).

The IDP vascularity had several interesting features. IDP are small (microscopic) dysplastic lesions often found in clusters. As shown in Figure 3, fields of IDP contain an extraordinary number of vessels, and among fields, the vascularity/epithelial areas are fairly consistent. However, Figure 4 shows that vascularity among individual IDPs varies enormously, indicating that at this stage a few IDPs have markedly increased angiogenic potentials relative to the rest. This angiogenic potential is actually greater than that of CIS. Therefore, in this system this angiogenic switch precedes epithelial expansion to CIS.

This early switch on of angiogenesis is a feature of other DMBA-induced pathologies. In the DMBA hamster pouch carcinogenesis model system, angiogenesis is found among populations of dysplastic keratinocytes, well before tumor formation occurs (Polverini and Solt, 1988). Isolated keratinocytes from this system are able to induce endothelial cell migration in vitro.

Our data show that HP are not more vascular than normal epithelium from DMBA-treated rats. These data are consistent with the concept that HP are unrelated to the genetic pathway leading to CIS and INV. Because tubular adenomas, which are the end result of this hyperplastic pathway, did not occur until beyond 3 months post-DMBA, we were not able to evaluate the vascularity of these end stage tumors.

Finally, we asked whether the changes in vascularity observed by morphometry were due to soluble factors derived from the epithelium or immediately adjacent stroma. The fact that the vasculature in DMBA-treated animals was more proliferative, stage for stage, is consistent with the idea that angiogenesis is occurring, although we cannot rule out the possibility that tissue remodeling also plays a role. At least by the stage of CIS, these tumors produce VEGF, a potent angiogenic growth factor, which correlates well with the robust dense vascularity seen around and within these tumors. The actual character of these vessels has been extensively examined by corrosion cast methodologies (Kaidoh et al, 1991). In these studies of DMBA-induced CIS, Kaidoh et al (1991) identified sinusoidal vessels, blind ends and bulbous outpouchings, consistent with both remodeling by the tumor cells and angiogenesis. Nakamura et al (1996) have also identified VEGF transcripts in DMBA-induced rat mammary tumors. Their data indicate that VEGF expression in these tumors is regulated by estrogen.

The RT-PCR data from isolated epithelial organoids gave inconsistent results, indicating that the angiogenic potential of normal epithelium from DMBAtreated rats is probably not due to VEGF. This is consistent with our immunologic studies in human breast tumorigenesis, which indicate that VEGF is not consistently present at high levels until the stage of CIS (Heffelfinger et al, 1999).

Our data contradicts one earlier study in which the angiogenic capacity of mammary tissues from DMBAand NMU-treated rats were examined in the rabbit iris model (Charnock-Jones et al, 1993). In this study $75 \%$ of primary tumors were angiogenic (consistent with our data) and became even more angiogenic after multiple generations of subcutaneous transplantation. However, unlike our study, primary "normal" tissues and HP from carcinogen-treated rats were not angiogenic. These tissues eventually displayed an angiogenic phenotype only after multiple generations of passaging and outgrowth. We believe that we can identify a morphometric angiogenic phenotype in DMBA-treated normal epithelium because we are quantifying vessels adjacent to the epithelium. Along with tumorigenesis, as seen in multiple in vivo passages, there is an increase in epithelial volume. Therefore, the question becomes, is angiogenesis induced in these tissues simply because there is more epithelium, or on a per cell basis (determined by epithelial area) do these tissues actually have a greater angiogenic potential? Our data show that, indeed, DMBA induces an increased angiogenic potential on a per cell basis even in histologically normal tissue. However, because of the relatively small amount of epithelium in normal rat mammary tissue, these changes were not detectable in the iris assay. In our in vitro tubule formation assay, we used isolated epithelial organoids to optimize the possibility of measuring any angiogenic factors present. Indeed, we were able to 
confirm that organoids of normal epithelium from DMBA-treated rats are uniquely angiogenic.

In conclusion, like human breast pathology, the progression of DMBA-initiated tissues in the rat from normal epithelium to invasive carcinoma is associated with an angiogenic phenotype. Even non-progressing epithelium, such as that which is histologically normal three months post-DMBA, elicits neovascularization. Unlike human disease, in the DMBA rat model a subset of early proliferative lesions, such as IDP, are at least as angiogenic as invasive carcinomas. Finally, VEGF is produced by CIS and INV in the later stages of disease progression. These results indicate that DMBA-induced rat mammary neoplasms are an appropriate model for testing the dependence of disease progression on angiogenesis.

\section{Materials and Methods}

\section{DMBA Treatment and Tissue Isolation}

Virgin female Sprague-Dawley rats, 50-55 days old, were given $20 \mathrm{mg}$ DMBA (prepared at $20 \mathrm{mg} / \mathrm{ml}$ in sesame oil by stirring at room temperature in the dark) or an equal volume of sesame oil as a single intragastric gavage. Rats were palpated weekly for three months to detect the presence of mammary tumors. Fifty milligrams per kilogram BrdU was administered ip to all animals at 24 and 12 hours before they were killed (3 months post-DMBA) in order to determine the proliferation index of the vasculature. Mammary tissue from the cervico-thoracic glands and the abdominalinguinal glands on each side were dissected away from the dermis and adjacent skeletal muscle and pooled for analysis. Tissue for histologic examination was fixed 12 to 24 hours in $10 \%$ buffered formalin. After fixation the mammary tissue was embedded in paraffin, sectioned, and stained with hematoxylin and eosin (H\&E). Gross tumors and hyperplastic lesions identified under the dissecting microscope were separated from normal tissue by dissection and processed separately. Pathologic diagnoses were according to Russo et al (1989). INV were defined as single cells and small clusters of malignant epithelium invading stromal space, adipose tissue, or skeletal muscle outside the confines of a basement membrane. All cases of INV were confirmed with cytokeratin immunohistochemistry.

\section{Isolation of Organoids}

Mammary glands were finely minced using scalpels and digested overnight at $37^{\circ} \mathrm{C}$ in $100 \mathrm{U} / \mathrm{ml}$ collagenase (Life Technologies, Gaithersburg, Maryland), 150 $\mathrm{U} / \mathrm{ml}$ hyaluronidase (Sigma, St. Louis, Missouri) in DMEM/F12 (Life Technologies, Gaithersburg, Maryland). The next day epithelial organoids, digested free of adjacent connective tissue were isolated by sedimentation on the benchtop. Sedimented organoids were washed free of contaminating skeletal muscle and vascular fragments by repeated suspension and resedimentation in DMEM/F12. Purity of each preparation was ascertained by inspection under the micro- scope. Individual ducts and lobules were readily identified using Hoffman optics.

\section{Immunohistochemistry}

Immunohistochemistry was performed using the Ventana 320ES automatic immunostaining system. This instrument adds the primary and secondary antibodies with appropriate intermediate washes in situ and counterstains the sections with hematoxylin using a barcode-driven protocol. For the monoclonal primaries, rat adsorbed anti-mouse (Vector Laboratories, Burlingame, California) was used as the secondary antibody at 1:50. Pre-immune rabbit IgG or isotypespecific mouse IgG added in place of the primary antibody were used as the negative controls. Histologic sections were double stained by immunohistochemistry for von Willebrand factor (polyclonal; DAKO, Carpenteria, California) and BrdU (clone 3D9, which was a gift from Dr. Azra Raza, Rush Presbyterian Hospital, Chicago, Illinois). Cytokeratin (clone C-11, Lab Vision, Fremont, California) staining was performed on tumors that were potentially invasive.

\section{Morphometry}

Images were captured using an Olympus BH-2 microscope attached to an Iris workstation (Silicon Graphics, Inc., Mountainview, California). The area of vessels was quantified relative to the total area of the duct/ lobule or tumor using Image Pro Plus (Media Cybernetics, Silver Springs, Maryland). Briefly, RGB image channels were separated for greater clarity of color determination. Vascular area (brown) was identified via the computer. The operator then tagged the subset of vessels with BrdU positive nuclei (blue). Epithelium of each histologic type was outlined by the operator and total area determined. Vessels that were "adjacent" to a particular epithelial unit, such as a duct, lobule, or type of pathology, remained associated with an epithelial area during analysis. "Adjacent" was defined as within the immediately adjacent stroma. For normal epithelium, HP, and IDPs the associated vessels were clearly identifiable and always within $50 \mu \mathrm{m}$ of the epithelial basement membrane. For CIS and invasive lesions, the stromal area was larger. However, the associated vascularity was also well defined, being no more than $350 \mu \mathrm{m}$ from the epithelial edge. To be certain that we were including an appropriate area around each lesion, in addition, all vessels within a $10 \times$ objective field were measured relative to the total epithelial area within the field.

\section{Tubule Formation}

Freshly isolated organoids (see "Isolation of Organoids," above) or tumor pieces of organoid size were placed on the surface layer of a collagen type I sandwich $2 \mathrm{mg} / \mathrm{ml}$ (Becton Dickinson, Bedford, Massachusetts) in which $3 \times 10^{4}$ human umbilical vein endothelial cells (HUVEC) (Clonetics, San Diego, California) per well had been cultured overnight in a 48 well dish. Individual organoids were selected under the microscope from the pool of 
digested products to assure the absence of stromal elements and an even distribution of epithelium among the wells. Seven hours later, 3-[4,5-Dimethylthiazole-2yl]-2,5-diphenyltetrazolium bromide (MTT) (1 $\mathrm{mg} / \mathrm{ml}$ ) (Sigma) was added. This vital dye stains the endothelium and mammary tissue a deep blue. Two hours later the wells were rinsed and fixed in $10 \%$ formalin. Tissues were removed and checked for viability and equality of distribution among replicate wells by MTT staining. Images of each well were captured using a VE-1000 camera (Dage-MTI) on an Olympus IMT-2 microscope and analyzed using Image Pro Plus image analysis software (Media Cybernetics) for total area and length of tubules. All conditions were assayed in quadruplicate and compared with media alone and media with 50 $\mathrm{ng} / \mathrm{ml}$ basic fibroblast growth factor (bFGF) (Becton Dickinson). In addition, tissues were compared with tubule formation induced by skeletal muscle fragments from control and DMBA-treated animals.

\section{RT-PCR}

Total cellular RNA was extracted from rat tumors using Tri-Reagent (Molecular Research Center, Cincinnati, Ohio). RNA was reverse transcribed using random oligonucleotides (cDNA Cycle Kit, Invitrogen, Carlsbad, California). Positive control RNA from rat uterus was a gift from Dr. Nira Ben Jonathan, University of Cincinnati, Cincinnati, Ohio. PCR primers were derived from the published rat sequence (Conn et al, 1990) and are as follows:

5' sense: GCTCTCTTGGGTGCACTGGA

3' antisense: CACCGCCTTGGCTTGTCACA.

These primers detect VEGF splice variants of 635 , 563, and $431 \mathrm{bp}$. Internal control primers for ribosomal protein L19, which produces a band at $333 \mathrm{bp}$, are as follows:

sense: TGCTCCATGAGAATCCGCTTG

antisense: CGAAATCGCCAATGCCAACTC.

The PCR reaction mixture utilized reagents from Life Technologies, except as noted: $5 \mu \mathrm{l} 10 \times$ buffer, $2 \mu \mathrm{l}$ $50 \mathrm{~mm} \mathrm{MgCl}$, $1 \mu \mathrm{l} 10$ nM dNTP (Invitrogen), 50 pmoles VEGF primers or 15 pmoles RPL19 primers, $4 \mu \mathrm{g}$ template DNA, $0.5 \mu \mathrm{l} 0.1 \mathrm{M}$ DTT, and 1 Unit Taq polymerase. Each reaction began with a 2-minute denaturation at $94^{\circ} \mathrm{C}$, followed by 35 cycles of a 30 -seconds denaturation $\left(94^{\circ} \mathrm{C}\right)$, a 30 -second annealing $\left(57^{\circ} \mathrm{C}\right)$, and a 30 -second extension $\left(72^{\circ} \mathrm{C}\right)$. A final 5 -minute extension at $72^{\circ} \mathrm{C}$ was also included. Of each reaction, $5 \mu \mathrm{l}$ was analyzed by polyacrylamide gel electrophoresis on a $5 \%$ acrylamide gel in $89 \mathrm{~mm}$ Tris-borate, $\mathrm{pH}$ 8.3, 2 mм EDTA.

\section{Statistics}

Mean values from the morphometric analyses and the tubule formation assays were compared within each experimental group by one way analysis of variance (ANOVA) and Dunnett's comparison test, $\alpha=0.05$ (SigmaStat, SPSS Science, Chicago, Illinois).

\section{Acknowledgements}

The authors would like to thank Anita Reaves and Janice Armstrong for their histologic services. We also thank Dr. Jose Russo for his advice on categorizing DMBA-induced mammary pathologies. Finally, we thank the Ruth Lyons Foundation and The Greater Cincinnati Foundation for their financial support of these studies.

\section{References}

Brem SS, Gullino PM, and Medina D (1977). Angiogenesis: A marker for neoplastic transformation of mammary papillary hyperplasia. Science 195:880-881.

Charnock-Jones SD, Sharkey AM, Rajput-Williams J, Burch D, Schodield JP, Fountain SA, Boocock CA, and Smith SK (1993). Identification of localization of alternately spliced mRNAs for vascular endothelial growth factor in human uterus and estrogen regulation in endometrial carcinoma cell lines. Biol Reprod 48:1120-1128.

Conn F, Bayne ML, Soderman DD Kwok PW, Sullivan KA, Palisi TM, Hope DA, and Thomas KA (1990). Amino acid and cDNA sequences of a vascular endothelial cell mitogen that is homologous to platelet-derived growth factor. Proc Natl Acad Sci USA 87:2628-2632.

Deng G, Lu Y, Zlotnikov G, Thor AD, and Smith HS (1996). Loss of heterozygosity in normal tissue adjacent to breast carcinomas. Science 274:2057-2059.

Dupont WD and Page DL (1985). Risk factors for breast cancer in women with proliferative breast disease. $\mathrm{N}$ Engl J Med 312:146-151.

Folkman J, Watson K, Ingber D, and Hanahan D (1989). Induction of angiogenesis during the transition from hyperplasia to neoplasia. Nature 339:58-61.

Fregene TS, Kellogg CM, and Pienta KJ (1994). Microvessel quantification as a measure of angiogenic activity in benign breast tissues lesions: A marker for precancerous disease? Internatl J Oncol 4:1199-1202.

Gimbrone MA and Gullino PM (1976). Neovascularization induced by intraocular xenografts of normal, preneoplastic, and neoplastic mouse mammary tissues. J Natl Cancer Institute 56:305-318.

Heffelfinger SC, Miller MA, Yassin R, and Gear R (In press, 1999). Angiogenic growth factors in pre-invasive breast disease. Clinical Cancer Res.

Heffelfinger SC, Yassin R, and Miller MA (1996). Lower E: Vascularity of proliferative breast disease and carcinoma in situ correlates with histologic features. Clinical Cancer Res 2:1873-1878.

Jensen HM, Chen I, DeVault MR, and Lewis AE (1982). Angiogenesis induced by "normal" human breast tissue: A probable marker for precancer. Science 218:293-294.

Kaidoh T, Yasugi T, and UeharaY (1991). The microvasculature of the 7:12-dimethylbenz(a)anthracene (DMBA)-induced rat mammary tumour. Virchows Archiv A Pathol Anat 418: 111-117.

Kandel J, Bossy-Wetzel E, Radnanyi F, Klagsbrun M, Folkman J, and Hanahan D (1991). Neovascularization is associated with a switch to the export of bFGF in the multistep development of fibrosarcoma. Cell 66:1095-1104. 
Larson PS, de las Morenas A, Cupples LA, Huang K, and Rosenberg CL (1998). Genetically abnormal clones in histologically normal breast tissue. Am J Pathol 152:1591-1598.

Nakamura J, Savinov A, Lu Q, and Brodie A (1996). Estrogen regulates vascular endothelial growth/permeability factor expression in 7:12 dimethylbenz(a)anthracene-induced rat mammary tumors. Endocrinology 137:5589-5596.

Ottinetti A and Sapino A (1988). Morphometric evaluation of microvessels surrounding hyperplastic and neoplastic mammary lesions. Breast Cancer Res and Treatment 11:241-248.

Polverini PJ and Solt DB (1988). Expression of the angiogenic phenotype by a subpopulation of keratinocytes derived from 7:12-dimehtylbenz[a]anthracene-initiated hamster buccal pouch epithelium. Carcinogenesis 9:117-122.
Russo J and Russo IH (1987). Biology of disease: Biological and molecular bases of mammary carcinogenesis. Lab Invest 57:112-137.

Russo J and Russo IH (1996). Experimentally induced mammary tumors in rats. Breast Cancer Res Treat 39:7-20.

Russo J, Russo IH, van Zwieten MJ, Rogers AE, and Gusterson BA (1989). Classification of neoplastic and nonneoplastic lesions of the rat mammary gland. In: Integument and Mammary Glands. TC Jones, U Mohr, and RD Hunt, editors. Berlin: Springer-Verlag, 275-304.

Smith-McCune KK and Weidner N (1994). Demonstration and characterization of the angiogenic properties of cervical dysplasia. Cancer Res 54:800-804. 